\title{
Cell Sheets: From Delivery Tool to a Model of Guided Regeneration
}

\section{Author list:}

Peter P. Nimiritsky ${ }^{1,2}$,

Roman Yu. Eremichev ${ }^{1}$,

Natalia A. Alexandrushkina ${ }^{1,2 \#}$, MD

Anastasia Yu. Efimenko ${ }^{1,2}, \mathrm{MD}, \mathrm{PhD}$

Vsevelod A. Tkachuk ${ }^{1-3}$, DSc

Pavel I. Makarevich ${ }^{* 1,2}, \mathrm{MD}, \mathrm{PhD}$

\section{Author affiliations}

1 - Institute for Regenerative Medicine, Medical research and education center, Lomonosov Moscow State University, Moscow, Russia

2 - Faculty of Medicine, Medical research and education center, Lomonosov Moscow State University, Moscow, Russia

3 - Laboratory of Molecular Endocrinology, National medical research center of cardiology, Moscow, Russia

* corresponding author: pmakarevich@mc.msu.ru

\# - equal contribution 


\begin{abstract}
Regeneration is a fundamental perpetual tissue process much attributed to functions of adult stem cell. In last decades delivery of adult stem cells to restore tissue structure is widely adopted in regenerative medicine. However, delivery of stem cells can not complete the task of human body regeneration effectively as far as cells need a basis to form a tissue. Certain improvement can be achieved when cells are organized to cells sheets (CS) - primitive tissue-engineered structures comprising of viable cells and ECM proteins. This led us to an assumption that after damage an intermediate feeder is structure formed as a ground for future tissue resulting in gradients of stimuli required to attract other cells (vascular, neural, epithelial, parenchyma etc.) in an organized manner.

This feeder acquires increasing heterogeneity and becomes a guiding feeder that provides basic level of organization to increase complexity of structure up to normal tissue. We also highlight potential role of adult mesenchymal stromal cells (MSC) as the main source of guiding feeder and support it by our recent knowledge of their biological function.

Finally, we assume that CS known to be a feasible delivery tool in therapeutics resemble guiding feeder and show our own data indicating properties of MSC-based CS. Furthermore, this concept shifts our understanding of therapeutic mechanism underlying a widely known delivery method for regenerative medicine.
\end{abstract}

Keywords: stem cell, stromal cell, mesenchymal stromal cell, regeneration, histogenesis, cell sheet, cell delivery 


\section{Introduction: regeneration as a tissue process of guided} organization

Living organisms possess an amazing ability to temporarily avoid perpetual increase of entropy stipulated by the Second law of thermodynamics and, thus, unavoidable destruction (which eventually occurs). This depends on another ability of living systems to utilize energy of the outer world and reduce internal entropy to keep their form/structure (e.g. to maintain chemical bonds and electrostatic interactions) which applies to all levels of organization - from single cell to tissue to whole organism [1].

Obviously, living organisms do not violate the Second law of thermodynamics, but manage to keep a balance between energy-dependent synthesis and spontaneous destruction constantly "re-creating" structures instead of those "destroyed by entropy". This leads us to a rather mechanistic conclusion that a living system exists in homeostasis due to permanent renewal triggered by a fundamental law of nature $[2,3]$.

This law also underlies recovery from a much more intense and less fundamental factor - namely damage. Successful regeneration after destruction of tissue down to molecular level requires "recreation" of native structure [4]. This can be achieved by moving down the path from chaos of initial stages immediately after damage to formation and progressive complication of connections between synthesized elements eventually increasing order in the system and, thus, reducing entropy $[5,6]$.

These nature-given mechanisms of human body support and repair after damage are employed by regenerative medicine which up to date relies on adult stem cell intrinsic potential to renew, differentiate and restore tissue structure and ultimately - function. In this paradigm our understanding of therapeutic regeneration in human body is "stem cell-centristic" portraying adult stem cells as regenerative "commando engineers" who can handle the whole operation on their own - from deployment to rebuilding functional tissue or organ once delivered viable and in sufficient amounts. 
Accumulated data suggests that regenerative potential of adult stem cell and outcome of its activation is defined by a combination of intrinsic abilities and conditions of environment that control, direct and support finely balanced stages of progenitor maturation and stem cell renewal [7]. Indeed, since Raymond Schofield's seminal paper [8] cell biology has adopted the concept of stem cell as a "nonautonomous" unit depending on its environment (namely, the "niche"). Critical role of environment has also been supported by investigation focused on molecular and cellular processes that drive tissue development and postnatal regeneration in primitive animals that show marvelous abilities to rebuild body parts or even the whole organism (e.g. planarians or starfish). Even in these creatures "isolated" adult stem cells lack ability to drive full-scale regeneration and require certain volume of tissue with intact interactions between elements [9].

This hypothesis article suggests a view on human body regeneration pinpointing the following points:

1. Early stages of regeneration result in formation of a stromal cell feeder that serves a basis for future increase of complexity during tissue formation;

2. Increase of complexity in the feeder results in tissue-like interactions between cells and heterogeneous structure and gradients of stimuli that play a potential guiding role for other cell types (guiding feeder);

3. Adult mesenchymal stromal cells (MSC) are the most prominent candidate cell type as a main producer of guiding feeder.

Finally, we suggest a model object for ex vivo study of MSC-derived guiding feeder - namely cell sheets widely considered as a delivery tool to avoid cell death due to apoptosis in suspended state and provide our initial experimental data. This moves our view on these constructs beyond a simple delivery platform suggests their use as a model of a critical physiologically occurring stage of tissue regeneration. We believe that cell sheets turn out to be a feasible model to understand mechanisms of tissue formation after damage and - potentially - utilize this knowledge to increase efficacy of stimulated human body repair which is the basis of regeneration. 


\section{Guiding feeder: stromal cells as organizers of regeneration}

\subsection{Tissue as an ecosystem and sequential recovery of its hierarchy during} regeneration

To form the basis of our concept we address classical principles that we need to keep in mind. First of all, proper function of an organ/tissue depends on 2 crucial factors:

1. Correct structure with normal topography of various cell types in their specific locations (compliance to an evolutionary-developed morphological "blueprint"). Physical stability of structure is supported by connections between elements: direct cell-to-cell contacts, interaction of cells with extracellular matrix (ECM) etc.

2. Integration of elements in a regulatory network tightly bound to the structure. Indeed, all cells in the tissue are and exchanging signals: soluble proteins, metabolites and electrochemical stimuli. Most of these signals distribute through the tissue and form gradients that result in different local microenvironments.

Crucial role of both listed features is a long-standing concept in physiology known as "unity of function and structure" [10]. Briefly speaking, to work effectively the tissue must have normal topography and undisturbed regulatory interactions between cells.

Furthermore, as the more specialized the cell becomes the more demanding it turns requiring accurate location and a definitive set of signals to maintain its correct function. Indeed, correct regulation and functional environment are extremely important for cells with finely tuned functions - e.g. adult stem/progenitor cells or parenchyma.

This brings us to a concept that tissue has certain hierarchy which can be portrayed as a pyramid where highly specialized cells reside on top but depend on the basis - a huge mass of other elements - endothelium, stromal cells, neural terminals etc. Described hierarchy existing in the tissue brings up allusions with biological ecosystems known as niches. 
The metaphor above is useful not only for explanation of how normal tissue is organized, but to describe tissue repair either. Severe damage to tissue results in loss of structure, and disruption of hierarchy with regulatory framework that has existed. In ecology this is similar to destruction of the ecosystem - e.g. a forest fire with complete burnout of vast areas to ashes and loss of all inhabitants of the ecosystem (Fig.1).

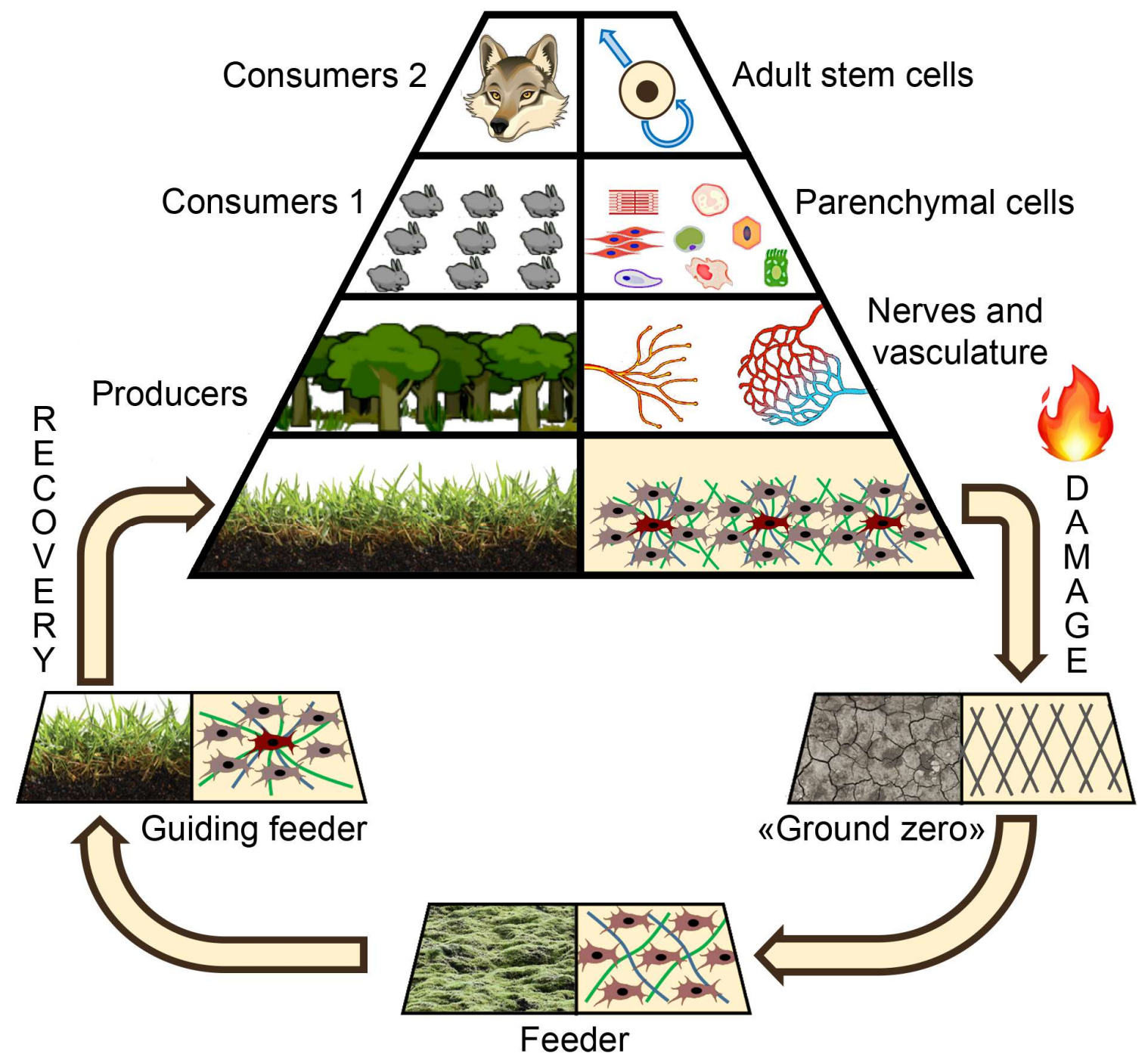

Figure 1. Tissue as an ecosystem in normal and regenerating states. The pyramid reflects structure and hierarchy of tissue elements in intact state. Arrows show sequence of stages required for tissue recovery and hierarchy restoration similar to events occurring after a forest ecosystem destruction by wildfire. 
Below, we summarize the most important points and show allusion between the regenerating tissue and the forest ecological niche. After a fire the site of catastrophe can not be immediately re-populated by the species from the top of food chain, but will undergo slow restoration of structure and hierarchy from the bottom starting with the most adaptive and viable species. When they generate a necessary trophic substrate then more demanding species can enter this system and populate it. Gradually the species diversity and biological capacity of entire ecosystem increases and both - structure and hierarchy is restored.

Extrapolated to the tissue this example illustrates the crucial role of early stages in tissue regeneration when a community of cells is formed to restore basic elements of microenvironment. Putative sequence of events generally corresponds in early stages generally corresponds to well-known reparative regeneration reaction:

1. Immediately after damage involved area is cleansed by inflammatory response. However, this phase leaves a "ground zero" with completely disrupted structure of the tissue down to the molecular level.

2. After cease of acute phase of inflammation stromal cells with a major fraction of MSC migrate to the damaged area and actively attenuating intensity of residual inflammation and proliferate.

3. When stromal cells are activated by local conditions after injury they produce basic elements of any tissue: ECM and trophic paracrine factors that attract and maintain the function of other cells. At this stage the ground is made for future restoration of tissue and stromal cells serve as a feeder.

However, such primitive feeder is not sufficient for structure recovery and formation of structural heterogeneity observed in mature tissue. Concept of a single cell type working as a regenerative scaffold or a priming quasi-tissue is actually not novel in zoology where we all are familiar with the salamander amputated limb regrowth. It is possible due to rapid formation of blastema which becomes the ground for a complex process of limb growth [11]. We hereby suggest that feeder 
undergoes a stage of maturation and believe it to be a tissue-like structure and apply the term "guiding". Both concepts arise from events occurring between the phase of basic tissue elements recovery and restoration of structure specialization:

4. ECM and paracrine factors produced by the feeder inevitably result in strengthening of intercellular interactions between the stromal cells. Eventually they self-organize and form areas of heterogeneity with differential production of structural factors and soluble proteins resulting in a gradient of stimuli. At this point the system is no longer just a sum of stromal cells, but has heterogeneous structure and forms hierarchy between elements.

5. As structural heterogeneity in feeder increases it results in signal gradient formation and, thus, specific areas of the feeder attract specific cell types. Some of them provide vascular nutrition and neural trophic rebuilding middle part of the "pyramid" (Fig. 1-2) making ground for the most specialized elements - stem/progenitor cells and parenchyma that stand on ttop of hierarchy. Frankly speaking, heterogeneity of feeder tissue serves a "guiding light" for further restoration of structure so we suggest the term guiding feeder for this system.

HOMOGENEITY HETEROGENEITY

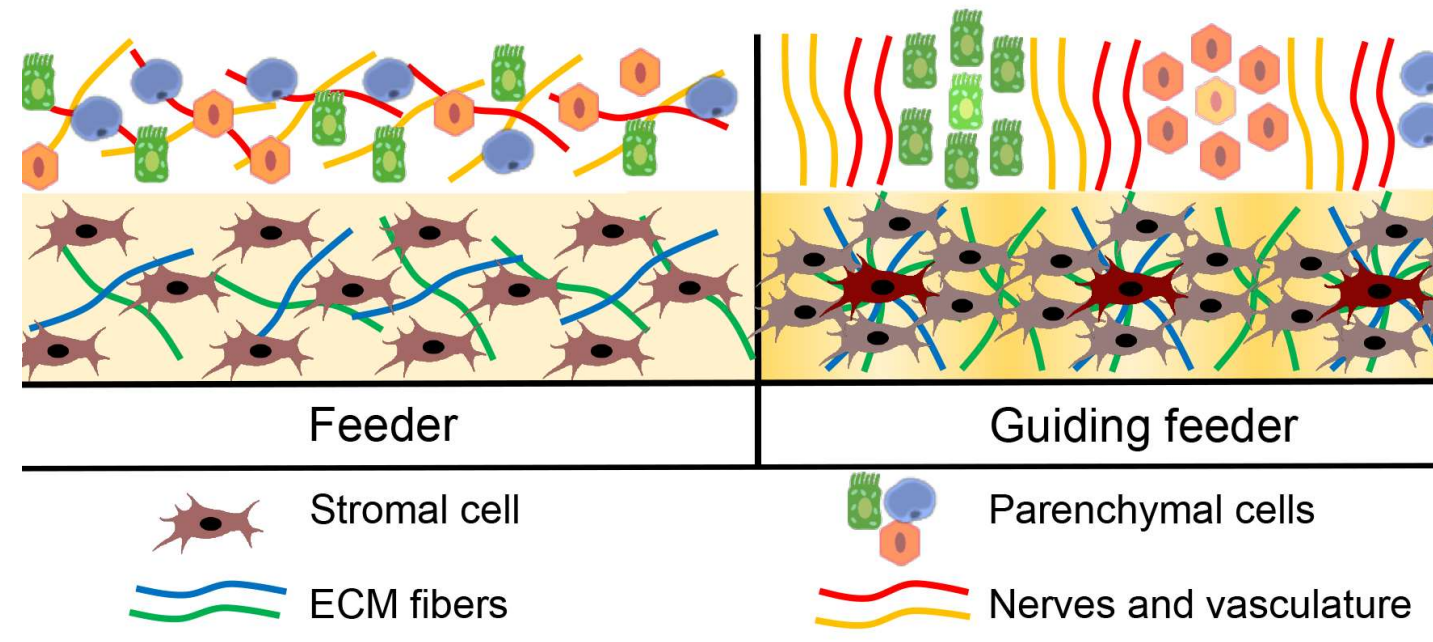

Figure 2. Heterogeneity of guiding feeder and complexity of the tissue. Left panel: initial stages of feeder formation lack hierarchy and gradients and, 
thus, fail to organize other cell types. Right panel: guiding feeder forms a distinct pattern of factors (ECM, secreted stimuli etc.) and hierarchy of elements eventually guiding other cell types to specific locations.

\subsection{Mesenchymal stromal cells as main structural elements of the guiding}

\section{feeder}

Among numerous cell types of human body (with over 250 types identified) we find MSC to be a prominent candidate the main cellular source of suggested guidance feeder. This assumption results from our understanding of their function in development, mature tissue maintenance and regeneration.

MSC are active participants of tissue formation since ontogenetic development [12]. Early stages of development are driven by embryonic stem cells' (ESCs) ability for self-organization yet later they rely on other cells that carry out "mapping" tissue microstructure by gradients of morphogenetic factors [13]. One of prominent examples in a very complex organ is crucial role of radial glia in development of central nervous system. Indeed, ablation of radial glia leads to severe brain malformations [14]. Furthermore, radial glia shares common characteristics with adult MSC (e.g. vimentin-positive intermediate filaments) and known to operate via active secretion of potent morphogens similarly to MSC. Thus, once certain cell type acts as structure organizer since development it may potentially retain this function in postnatal period for renewal and regeneration of tissue.

In postnatal phase MSC are located perivascular and present an ubiquitous component in majority of organs. In quiescent state they perform a "housekeeping" function: control vascular permeability, support ECM production and turnover, secrete growth factors and retain tissue architecture by creation of heterogeneous gradients of stimuli. This function extends up to local tissue progenitors and stem cells: intestinal crypt niche MSCs create a gradient of bone morphogenic proteins (BMP) that control resident stem cell fate and its differentiation [15]. However, this function of MSC is not limited to specific niches and in intact tissue MSC participate in formation of microenvironment for all cell types located there [16]. Thus, MSC 
are involved in maintaining of structure and cellular homeostasis of healthy tissue [17].

However, after damage MSC show prominent abilities that are prerequisites for their potential role of a main cellular source for the guiding feeder described above.

Table 1. MSC biological properties that support its role as a cellular source of guiding feeder

\begin{tabular}{|l|l|}
\hline \multicolumn{1}{|c|}{ MSC function } & \multicolumn{1}{c|}{ Reference } \\
\hline Ubiquitous location in tissues and organs & Bianco, et al. [18] \\
\hline Multipotency and high-proliferative potential & Pittenger, et al. [19] \\
\hline Survival under stress conditions and inflammation & $\begin{array}{l}\text { Lee, et al. [20] } \\
\text { Oh, et al. [21] }\end{array}$ \\
\hline $\begin{array}{l}\text { Immunomodulatory activity (reduction of } \\
\text { inflammation) }\end{array}$ & $\begin{array}{l}\text { Spaeth, et al. [22] } \\
\text { Bernardo and Fibbe [23] } \\
\text { Semedo, et al. [24] }\end{array}$ \\
\hline Active ECM production and turnover & Kalinina, et al. [25] \\
\hline Active pleiotropic secretome & $\begin{array}{l}\text { Rubina, et al. [26] } \\
\text { Makarevich, et al. [27] }\end{array}$ \\
\hline
\end{tabular}

In case of damage MSC are activated and migrate to the lesion site where they proliferate [28]. Their high proliferative potential resulted in excellent cultural properties of MSC especially under damage-modelling conditions: obviously, serum added to most culture media has certain similarity to wound exudate. To mitigate residual inflammation MSC secrete a wide range of anti-inflammatory factors and switch the tissue to regenerative state. Further, MSC produce ECM proteins, and factors of ECM turnover (matrix metalloproteases etc.), as well as chemoattractants and trophic paracrine factors for attraction and maintaining function of other cell types in the regenerating region. For example MSCs paracrine factors are directing 
the growth of blood vessels and nerves. Thus, we see that MSC possess all features required to produce the feeder.

However, the main part - the "guiding" function of the feeder seems completely elusive from our recent knowledge and depends on a complex and heterogeneous structure making it marginally unexplorable in vivo. However, in our numerous works focused on developing new stem cell delivery methods [27, 29, 30] we stumbled upon widely disregarded spontaneous heterogeneity in CS from MSC which hinted their use as a potential ex vivo model of suggested guiding feeder. Below we provide a brief overview to support choice of model object and provide our preliminary data.

\section{Potential ex vivo model of guiding feeder}

\subsection{Cell sheet technology: basics and application}

Cell Sheets (CS) are scaffold-free tissue-engineering constructs consisting of cells and ECM accumulated in cultural conditions. Formation of CS varies in time (3-14 days) and depends on cells proliferation, ECM and paracrine factors production resulting in a multilayered construct that readily detaches from the dish by different methods (treatment with EDTA solution [30], thermo-responsive coating [31] etc.).

This technology quickly found application in many areas of regenerative medicine, primarily due to advantages over routinely used direct injection. Injection of cells has drawbacks mainly related to massive cell death [32], as well as cell stress, loss of surface receptors, contacts and destruction of environment due to processing by proteolytic enzymes [33]. From this position, the use of cell sheets as a method of delivering cells in an intact state, with a native microenvironment, seems to be the most attractive. On the other hand, CS are not classically engineered tissue - they more resemble an extremely dense culture yet considered feasible when complex structure is not required: for example, to cover skin wounds or ulcers or to facilitate bone/cartilage defect healing [34,35]. Many preclinical and clinical studies using CS technology were aimed at treating diseases of the bones, cartilage, skin, 
urinary bladder, heart, blood vessels, esophagus, cornea etc [36]. Nevertheless, despite the practical significance of CS technology, the mechanisms underlying cell self-organization, ECM deposition and formation of intercellular contacts have not been studied.

\subsection{Using cell sheets as a model object to study guiding feeder properties}

MSC of different sources readily form CS and we lately have focused on experimental proof of their efficacy in numerous disorders ranging from wound healing to myocardial ischemia $[29,37,38]$. However, concept of MSC as a source of guiding feeder drew our attention to the fact that MSC-derived CS show vivid spontaneous heterogeneity despite being initially seeded from a relatively homogeneous cell population. Furthermore, this heterogeneity is formed after a certain period of time and brings up the "missing" link - formation of focal stimuli which we stress as a crucial factor of tissue organization and regeneration after damage. In this final part we focus on our preliminary data that reveal distinctive similar features of MSC-derived CS and putative guiding feeder described above. For details of experimental procedures please address supplementary Materials and methods online.

\subsubsection{Spontaneous formation of morphological heterogeneity}

During culture MSC form CS and then spontaneously show heterogeneity of cell density corresponding to putative stage of guiding feeder maturation. Two distinct compartments are formed: 1) compact multi-layered aggregates 300-500 microns wide with increased density of cells ("hills") surrounded by 2) areas with cell density similar to confluent MSC culture ("valleys"). Both of them are visible macroscopically in a dish (Figure 3, a) and can be discerned one from another under magnification (Figure 3, b and c).

Under normal culture conditions "hills" are formed as a result of selforganization of MSC in CS and time-lapse microscopy clearly showed dynamics of the process. This is an active process consisting of proliferation followed by joint 
coordinated contracting and movement of cells and their groups relative to each other (Supplementary video 1).
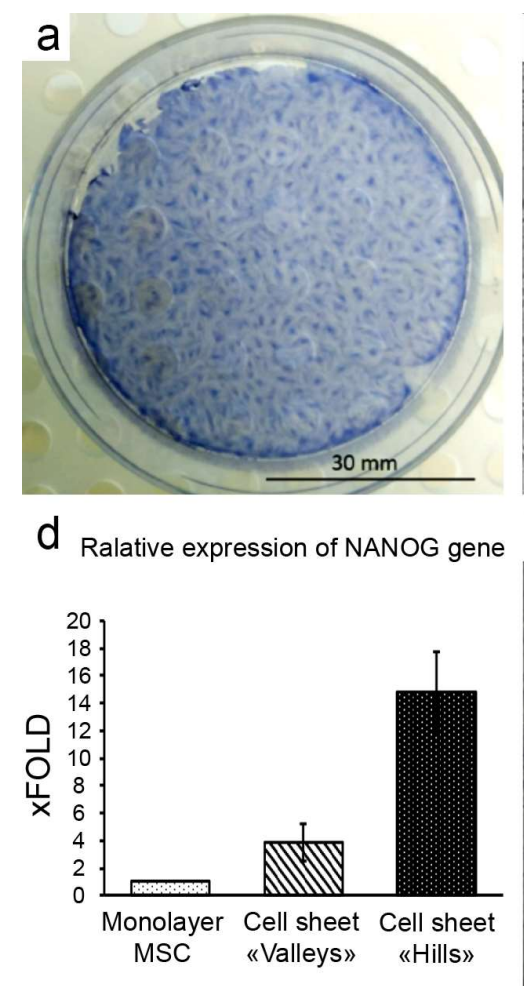
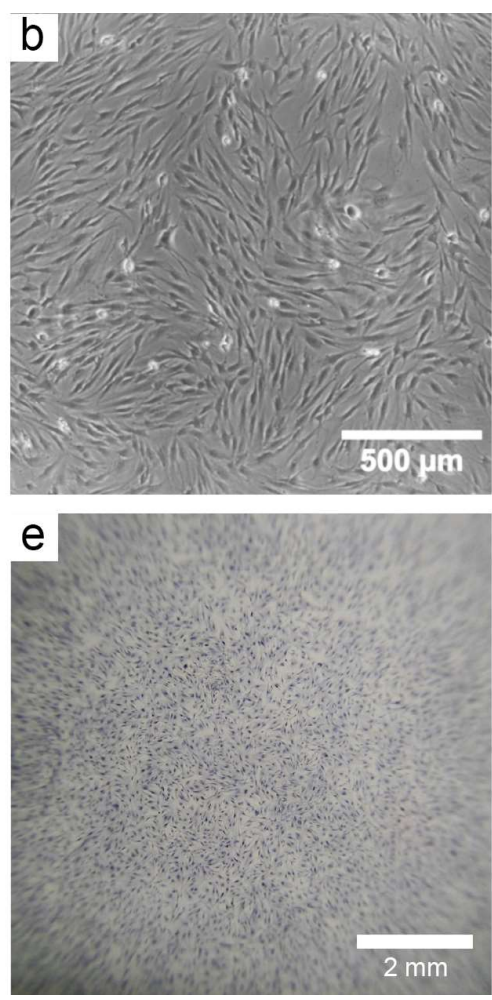
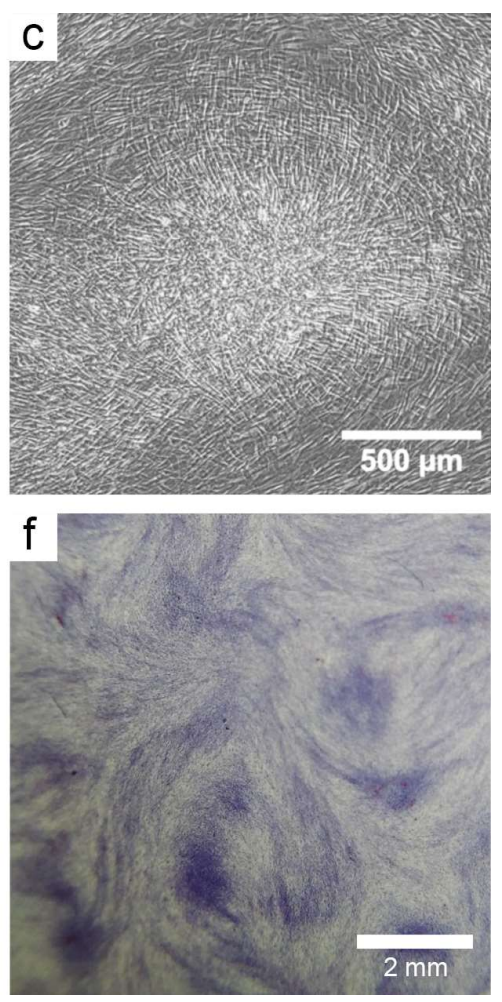

Figure 3. Self-organization of MSC in vitro results in formation of heterogeneous environment in cell sheets

a. Heterogeneous distribution of MSC in mature CS; hematoxylin staining;

b. Self-organization of MSC in CS results in formation of hills; hematoxylin staining;

c. Self-organization of MSC in CS results in formation of hills (snapshots from Supplementary video 1); microphotography, phase contrast.;

d. MSC subpopulations were picked by laser microdissection. MSC in the "hills" show increased expression of stemness-associated factor (NANOG); real-time qPCR $(n=3)$; data expressed as mean \pm standard deviation are shown.

3.2.2 Cell density distribution heterogeneity correlates with heterogeneity of MSC subpopulations 
Spontaneous formation of feeder heterogeneity ("hills and valleys") may have functional outcome. Namely may provide more or less favorable conditions for different MSC subpopulations or shift MSC differentiation status due to environment changes. Using laser microdissection we compared relative expression of stemness-related gene $N A N O G$ in MSC from different microenvironments. We compared monolayer culture to separately studied "valleys" and "hills" laserdissected from a CS imitating mature guiding feeder. We found that "hills" showed significantly increased expression of $N A N O G$ than monolayer or "valleys" of same CS (Figure 3, d). It suggested that guiding feeder (or CS) heterogeneity results in either sorting of different cells to different compartments of the construct or local shift of stemness - in particular, higher $N A N O G$ expression. In any case, this set of data is an evidence that structural heterogeneity is accompanied by changes of MSC status in situ which resembles primitive hierarchy and putatively related to focal gradients of stimuli in the guiding feeder.

\subsubsection{Cell distribution heterogeneity correlates with heterogeneity of ECM}

\section{distribution}

Guiding function of the feeder relies much on structural proteins of ECM that should form differential distribution of them in CS to create local microenvironments. We studied the features of 2 crucial ECM proteins - fibronectin and laminin CS (Fig. 4). In our experiment "hills" and "valleys" showed different patterns of fibronectin packing. Curiously laminin deposition was detectable only in "hills" and monolayer no acquirable signal detected. Since laminin is an ECM protein involved in cooperative interaction of stroma with endothelium and epithelium we assume that heterogeneity of laminin distribution may have functional meaning and, thus, indicates significant changes on tissue environment in the "hills". Thus, differential distribution of laminin in CS can be the basis of increased complexity of that is a function of the guiding feeder. 


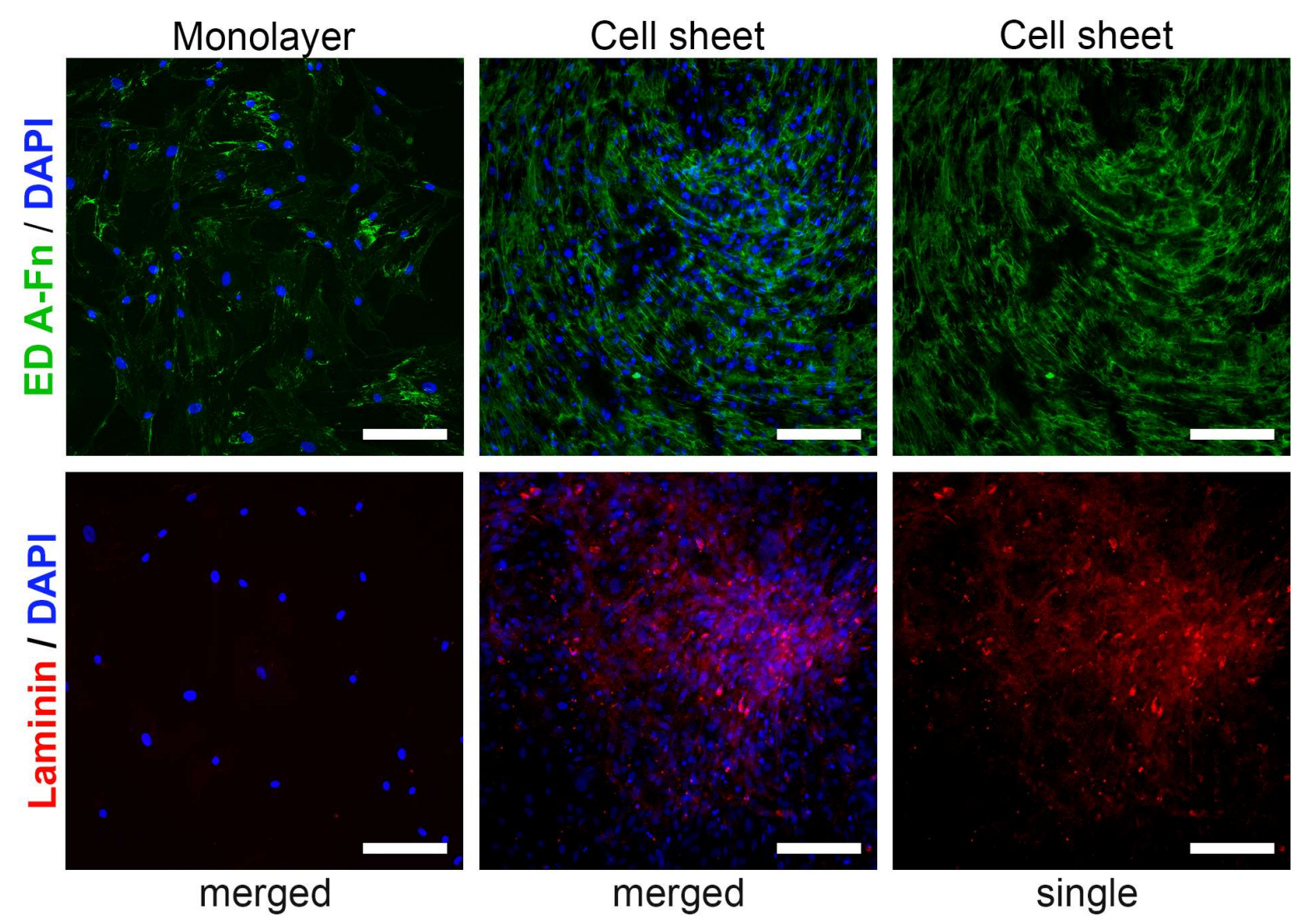

Figure 4. Heterogeneity of ECM deposition in CS compared to monolayer. During formation of cell sheet MSC organise EDA-Fibronectin in regular network of fibres (upper row); laminin shows a distinct pattern of deposition primarily in "hills", but not in "valleys" creating foci of heterogeneity in CS. Immunofluorescent microscopy, nuclei are stained by DAPI.

\section{Conclusions}

Presented concept of guiding feeder is a subject for discussion; however, it drives us to shift the focus from "cell-censtristic" approach in regenerative medicine to "tissue-centristic" view on human body regeneration after damage. To conclude, we shall summarize the main points of the hypothesis we promote by this article:

1. Regeneration as a process is characterized by increase of heterogeneity and complexity of intercellular communications that define tissue topography and organization to ensure function;

2. Existing hierarchy of tissue elements results in hierarchy of regeneration which resembles order of ecological niche recovery. 
3. Initial stages of regeneration are potentially stem cell-independent, but they lay ground of a feeder enriched with ECM and secreted factors;

4. Self-organization of feeder tissue results in formation of focal stimuli the distinctive feature of guiding feeder - becoming the basis of further successful regeneration

5. Mesenchymal stromal cells (MSC) are a putative source of the guiding feeder tissue and its components due to their ubiquitous location and biological properties

To further elucidate the role and structure of suggested MSC-based guiding feeder we propose ex vivo use of a feasible object - namely cell sheets. It has long been known that 3D approaches demonstrate more physiological conditions and similarity with native tissue than 2D monolayer [39]. We believe that CS can be considered not only as a mean of therapeutic use, but also as an object for investigation as far as it mimics key features of MSC-based guiding feeder as we stipulated them in our hypothesis. Previously observed positive effects of therapy by CS can be attributed not only effective delivery of viable cells and presence of ECM, but also rendered by organization of cells, matrix and secreted factors that build up a tissue-like regulatory network and form spontaneous heterogeneity we observed in vitro in CS. 


\section{References}

1. Lehninger, A. L.; Nelson, D. L.; Cox, M. M., Principles of biochemistry. 2nd ed.; Worth Publishers: New York, NY, 1993; p xli, 1013, 77 p.

2. Koshland, D. E., Jr., Special essay. The seven pillars of life. Science 2002, 295, (5563), 2215-6.

3. Zotin, A. I.; Zotina, R. S., Thermodynamic aspects of developmental biology. $J$ Theor Biol 1967, 17, (1), 57-75.

4. Agata, K.; Saito, Y.; Nakajima, E., Unifying principles of regeneration I: Epimorphosis versus morphallaxis. Dev Growth Differ 2007, 49, (2), 73-8.

5. Rinkevich, B.; Rinkevich, Y., The "Stars and Stripes" Metaphor for Animal Regeneration-Elucidating Two Fundamental Strategies along a Continuum. Cells 2012, 2, (1), 1-18.

6. Sena, G.; Birnbaum, K. D., Built to rebuild: in search of organizing principles in plant regeneration. Curr Opin Genet Dev 2010, 20, (4), 460-5.

7. Morrison, S. J.; Spradling, A. C., Stem cells and niches: mechanisms that promote stem cell maintenance throughout life. Cell 2008, 132, (4), 598611.

8. Schofield, R., The relationship between the spleen colony-forming cell and the haemopoietic stem cell. Blood Cells 1978, 4, (1-2), 7-25.

9. Tanaka, E. M.; Reddien, P. W., The cellular basis for animal regeneration. Dev Cell 2011, 21, (1), 172-85.

10. Saladin, K., Anatomy and physiology: The unity of form and function. 2007. Ohio: McGraw-Hill 2010.

11. McCusker, C. D.; Gardiner, D. M., Positional Information Is Reprogrammed in Blastema Cells of the Regenerating Limb of the Axolotl (Ambystoma mexicanum). PLoS ONE 2013, 8, (9).

12. Gilbert, S. F.; Barresi, M., DEVELOPMENTAL BIOLOGY, 2016. American Journal of Medical Genetics Part A 2017, 173, (5), 1430-1430. 
13. Li, N.; Xie, T.; Sun, Y., Towards organogenesis and morphogenesis in vitro: harnessing engineered microenvironment and autonomous behaviors of pluripotent stem cells. Integrative Biology 2018, 10, (10), 574-586.

14. Barry, D. S.; Pakan, J. M.; McDermott, K. W., Radial glial cells: key organisers in CNS development. The international journal of biochemistry \& cell biology 2014, 46, 76-79.

15. Kosinski, C.; Li, V. S.; Chan, A. S.; Zhang, J.; Ho, C.; Tsui, W. Y.; Chan, T. L.; Mifflin, R. C.; Powell, D. W.; Yuen, S. T., Gene expression patterns of human colon tops and basal crypts and BMP antagonists as intestinal stem cell niche factors. Proceedings of the National Academy of Sciences 2007, 104, (39), 15418-15423.

16. Kfoury, Y.; Scadden, D. T., Mesenchymal cell contributions to the stem cell niche. Cell stem cell 2015, 16, (3), 239-253.

17. Klimczak, A.; Kozlowska, U., Mesenchymal stromal cells and tissuespecific progenitor cells: their role in tissue homeostasis. Stem cells international 2016, 2016.

18. Bianco, P.; Cao, X.; Frenette, P. S.; Mao, J. J.; Robey, P. G.; Simmons, P. J.; Wang, C.-Y., The meaning, the sense and the significance: translating the science of mesenchymal stem cells into medicine. Nature medicine 2013, $19,(1), 35$.

19. Pittenger, M. F.; Mackay, A. M.; Beck, S. C.; Jaiswal, R. K.; Douglas, R.; Mosca, J. D.; Moorman, M. A.; Simonetti, D. W.; Craig, S.; Marshak, D. R., Multilineage potential of adult human mesenchymal stem cells. science 1999, 284, (5411), 143-147.

20. Lee, S.; Choi, E.; Cha, M.-J.; Hwang, K.-C., Cell adhesion and long-term survival of transplanted mesenchymal stem cells: a prerequisite for cell therapy. Oxidative medicine and cellular longevity 2015, 2015.

21. Oh, E. J.; Lee, H. W.; Kalimuthu, S.; Kim, T. J.; Kim, H. M.; Baek, S. H.; Zhu, L.; Oh, J. M.; Son, S. H.; Chung, H. Y., In vivo migration of 
mesenchymal stem cells to burn injury sites and their therapeutic effects in a living mouse model. Journal of Controlled Release 2018, 279, 79-88.

22. Spaeth, E.; Klopp, A.; Dembinski, J.; Andreeff, M.; Marini, F., Inflammation and tumor microenvironments: defining the migratory itinerary of mesenchymal stem cells. Gene therapy 2008, 15, (10), 730.

23. Bernardo, M. E.; Fibbe, W. E., Mesenchymal stromal cells: sensors and switchers of inflammation. Cell stem cell 2013, 13, (4), 392-402.

24. Semedo, P.; Palasio, C. G.; Oliveira, C. D.; Feitoza, C. Q.; Gonçalves, G. M.; Cenedeze, M. A.; Wang, P. M.; Teixeira, V. P.; Reis, M. A.; PachecoSilva, A., Early modulation of inflammation by mesenchymal stem cell after acute kidney injury. International immunopharmacology 2009, 9, (6), 677682 .

25. Kalinina, N.; Kharlampieva, D.; Loguinova, M.; Butenko, I.; Pobeguts, O.; Efimenko, A.; Ageeva, L.; Sharonov, G.; Ischenko, D.; Alekseev, D., Characterization of secretomes provides evidence for adipose-derived mesenchymal stromal cells subtypes. Stem cell research \& therapy 2015, 6, (1), 221.

26. Rubina, K.; Kalinina, N.; Efimenko, A.; Lopatina, T.; Melikhova, V.; Tsokolaeva, Z.; Sysoeva, V.; Tkachuk, V.; Parfyonova, Y., Adipose stromal cells stimulate angiogenesis via promoting progenitor cell differentiation, secretion of angiogenic factors, and enhancing vessel maturation. Tissue Engineering Part A 2009, 15, (8), 2039-2050.

27. Makarevich, P. I.; Boldyreva, M. A.; Gluhanyuk, E. V.; Efimenko, A. Y.; Dergilev, K. V.; Shevchenko, E. K.; Sharonov, G. V.; Gallinger, J. O.; Rodina, P. A.; Sarkisyan, S. S., Enhanced angiogenesis in ischemic skeletal muscle after transplantation of cell sheets from baculovirus-transduced adipose-derived stromal cells expressing VEGF165. Stem cell research \& therapy 2015, 6, (1), 204.

28. Caplan, A. I., Mesenchymal Stem Cells: Time to Change the Name! Stem Cells Transl Med 2017, 6, (6), 1445-1451. 
29. Dergilev, K.; Tsokolaeva, Z.; Makarevich, P.; Beloglazova, I.; Zubkova, E.; Boldyreva, M.; Ratner, E.; Dyikanov, D.; Menshikov, M.; Ovchinnikov, A.; Ageev, F.; Parfyonova, Y., C-Kit Cardiac Progenitor Cell Based Cell Sheet Improves Vascularization and Attenuates Cardiac Remodeling following Myocardial Infarction in Rats. Biomed Res Int 2018, 2018, 3536854.

30. Dergilev, K. V.; Makarevich, P. I.; Tsokolaeva, Z. I.; Boldyreva, M. A.; Beloglazova, I. B.; Zubkova, E. S.; Menshikov, M. Y.; Parfyonova, Y. V., Comparison of cardiac stem cell sheets detached by Versene solution and from thermoresponsive dishes reveals similar properties of constructs. Tissue Cell 2017, 49, (1), 64-71.

31. Okano, T.; Yamada, N.; Sakai, H.; Sakurai, Y., A novel recovery system for cultured cells using plasma-treated polystyrene dishes grafted with poly $(\mathrm{N}-$ isopropylacrylamide). J Biomed Mater Res 1993, 27, (10), 1243-51.

32. Aguado, B. A.; Mulyasasmita, W.; Su, J.; Lampe, K. J.; Heilshorn, S. C., Improving viability of stem cells during syringe needle flow through the design of hydrogel cell carriers. Tissue Eng Part A 2012, 18, (7-8), 806-15.

33. Kushida, A.; Yamato, M.; Isoi, Y.; Kikuchi, A.; Okano, T., A noninvasive transfer system for polarized renal tubule epithelial cell sheets using temperature-responsive culture dishes. Eur Cell Mater 2005, 10, 23-30; discussion 23-30.

34. Chen, L.; Xing, Q.; Zhai, Q.; Tahtinen, M.; Zhou, F.; Chen, L.; Xu, Y.; Qi, S.; Zhao, F., Pre-vascularization Enhances Therapeutic Effects of Human Mesenchymal Stem Cell Sheets in Full Thickness Skin Wound Repair. Theranostics 2017, 7, (1), 117-131.

35. Roh, J. L.; Lee, J.; Kim, E. H.; Shin, D., Plasticity of oral mucosal cell sheets for accelerated and scarless skin wound healing. Oral Oncol 2017, $75,81-88$.

36. Kirby, G. T. S.; Michelmore, A.; Smith, L. E.; Whittle, J. D.; Short, R. D., Cell sheets in cell therapies. Cytotherapy 2018, 20, (2), 169-180. 
37. Hsu, M. N.; Liao, H. T.; Li, K. C.; Chen, H. H.; Yen, T. C.; Makarevich, P.; Parfyonova, Y.; Hu, Y. C., Adipose-derived stem cell sheets functionalized by hybrid baculovirus for prolonged GDNF expression and improved nerve regeneration. Biomaterials 2017, 140, 189-200.

38. Makarevich, P. I.; Boldyreva, M. A.; Gluhanyuk, E. V.; Efimenko, A. Y.; Dergilev, K. V.; Shevchenko, E. K.; Sharonov, G. V.; Gallinger, J. O.; Rodina, P. A.; Sarkisyan, S. S.; Hu, Y. C.; Parfyonova, Y. V., Enhanced angiogenesis in ischemic skeletal muscle after transplantation of cell sheets from baculovirus-transduced adipose-derived stromal cells expressing VEGF165. Stem Cell Res Ther 2015, 6, 204.

39. Pampaloni, F.; Reynaud, E. G.; Stelzer, E. H., The third dimension bridges the gap between cell culture and live tissue. Nat Rev Mol Cell Biol 2007, 8, (10), 839-45. 